\title{
Bullous eruption in a five-month-old girl
}

\author{
Aida Khaled MD, Monia Kharfi MD, Becima Fazaa MD, Mohamed Ridha Kamoun MD
}

Previously published at www.cmaj.ca

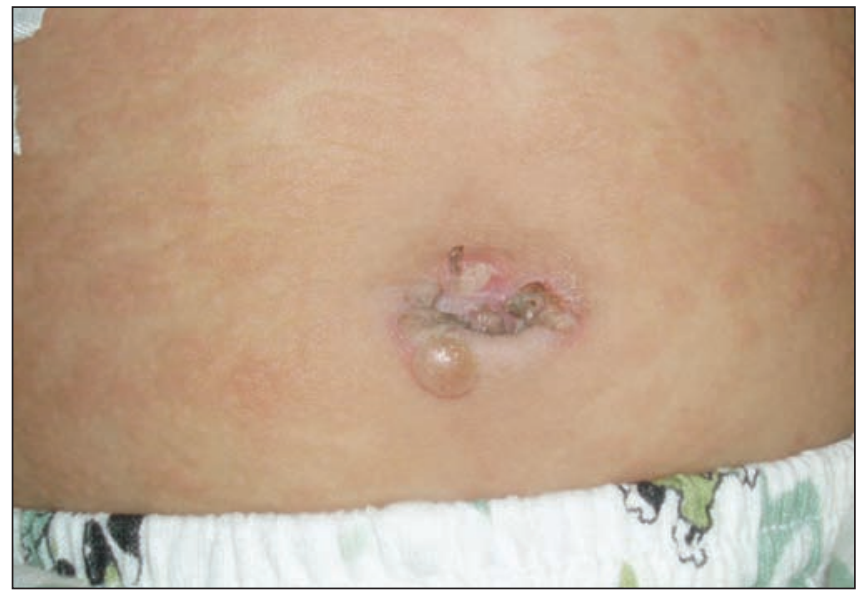

Figure 1: Urticarial papules with tense bullae on the abdomen and crusted omphalitis in a five-month-old girl.

\begin{abstract}
A five-month-old girl was referred to our department for an erythematous papular nonpruritic eruption that evolved into the formation of blisters on the trunk, hands and feet over a two-week period. Her medical history was unremarkable. She had received a combined polio, diphtheria, tetanus and pertussis vaccine and hepatitis $B$ vaccine four weeks before the onset of the cutaneous lesions. Her mother did not report any history of her own skin blistering during pregnancy. Physical examination showed many urticarial papules on the trunk and limbs with multiple vesicles and tense bullae on the abdomen, limbs and dorsum of the hands and feet (Figures 1, 2 and 3). A few vesicles extended onto the palms and soles. The mucous membrane was not involved. Findings on physical examination were otherwise normal. In particular, there was no fever. The infant was breast-feeding and growing normally.
\end{abstract}

\section{When faced with a situation like this, what should the physician do next?}

a. Swab the lesions for bacterial and viral cultures.

b. Initiate a sepsis workup and start antibiotics.

c. Do a skin biopsy and treat the lesions with chlorhexidine.

d. Prescribe corticosteroids.

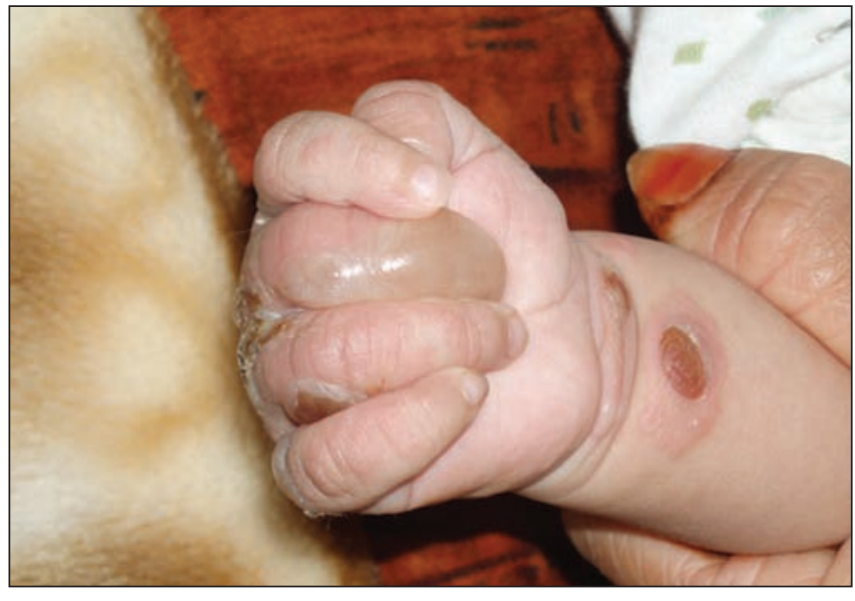

Figure 2: Urticarial papules with tense bullae on the hand of a five-month-old girl.

Because this infant had a bullous eruption without fever and was otherwise well, we suspected an autoimmune blistering disease. We did a skin biopsy (c) to confirm the diagnosis by histologic examination and direct immunofluorescence. We started localized treatment with chlorhexidine to prevent bacterial infection.

\section{What is your diagnosis?}
a. Linear immunoglobulin (IgA) dermatosis
b. Bullous impetigo
c. Bullous pemphigoid
d. Bullous mastocytosis
e. Dermatitis herpetiformis

In our patient, the results of histological examination of a cutaneous biopsy showed subepidermal bulla with abundant eosinophils in the papillary dermis. Direct immunofluorescence showed linear deposition of immunoglobulin G (IgG, faint deposits) and complement component 3 (C3, intense deposits) along the basement membrane zone leading to the

From the Department of Dermatology, Charles Nicolle Hospital, Tunis, Tunisia

CMAJ 2010. DOI:10.1503/cmaj.090867 


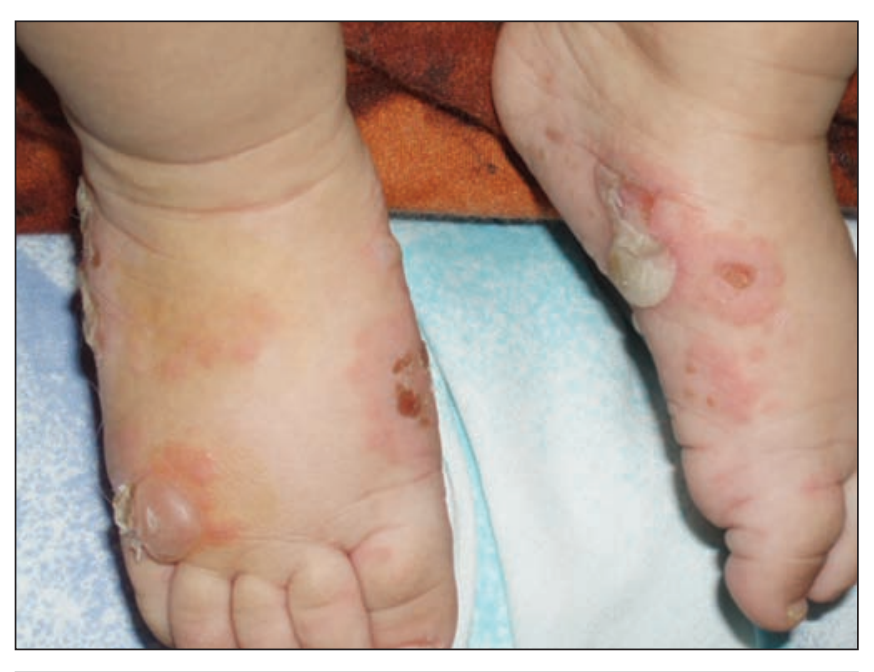

Figure 3: Urticarial papules with tense bullae on the feet of a five-month-old girl.

diagnosis of bullous pemphigoid (c). The girl was treated with topical steroid (clobetasol propionate) twice daily, which led to a rapid resolution of the skin lesions within two months. Within a two-year follow-up period, she had no relapse of the lesions, even after the topical steroids were stopped.

\section{Discussion}

Bullous pemphigoid is an acquired autoimmune subepidermal blistering disease that predominantly affects elderly people. Childhood bullous pemphigoid is rarely reported in the literature, but it constitutes the second most common acquired immunobullous disease of children, with linear IgA dermatosis being the most common. The precise incidence of bullous pemphigoid in children is unknown. In Israel, it was estimated at 2.36 per 100000 annually among infants under the age of one year. ${ }^{1}$ A recent review of the literature, which included 79 reported cases of childhood bullous pemphigoid, revealed two peaks of incidence: in the first year of life $(53 \%$, median age four months) and at the age of eight years (47\%), and a female predominance (F: 62\%, M: 38\%). ${ }^{1}$

As with our patient, there have been several previous reports of a temporal association of bullous pemphigoid with vaccination (e.g., hepatitis B, influenza). ${ }^{2,3}$ However, other data do not support the hypothesis of vaccination being an important trigger for severe bullous pemphigoid at a population level. ${ }^{2}$

\section{Clinical features}

Childhood bullous pemphigoid is clinically similar to adult bullous pemphigoid, with a bullous eruption made of tense vesicles and bullae of variable size reaching many centimetres in diameter. Blisters arise on urticarial papules, as observed in our patient. Pruritus is usually present and may precede the eruption by a few days or months. Although mucous membrane involvement is rare in adult bullous pemphigoid, it seems to be more frequent in children, especially with vulvar lesions occurring in girls aged eight years and more. ${ }^{1}$ Hand and feet involvement, as observed in our patient, is more frequently seen in infants.

\section{Diagnosis}

In 1991, Nemeth and colleagues defined the following diagnostic criteria for childhood bullous pemphigoid: the patient is younger than 18 years; the patient has bullous skin lesions, with or without mucous membrane involvement; there are characteristic histopathologic features of bullous pemphigoid (subepidermal bullae with variable amount of eosinophils); and direct immunofluorescence shows linear deposition of IgG and/or C3 at the basement membrane zone, or positive results of indirect immunofluorescence show IgG antibodies reacting with the basement membrane zone. ${ }^{4}$ The diagnosis of bullous pemphigoid is based on these histological and immunopathological criteria and, in some cases, on characterization of the target antigen. Indeed, as in adult bullous pemphigoid, antibodies against the NC16A domain of BP180 seem to be involved in the pathogenesis of the disease. ${ }^{5}$

\section{Differential diagnosis}

Childhood bullous pemphigoid may be confused with several other childhood bullous dermatoses, especially linear IgA dermatosis, bullous impetigo, dermatitis herpetiformis and bullous mastocytosis.

\section{Linear IgA dermatosis}

Linear IgA dermatosis is a rare acquired autoimmune subepidermal bullous disease characterized by continuous linear IgA deposits in the basement membrane zone, visualized on direct immunofluorescence microscopy. It is considered the most frequent immunobullous dermatosis among children and may be clinically confused with childhood bullous pemphigoid. The diagnosis of linear IgA dermatosis is made following demonstration of linear deposits of $\operatorname{IgA}$ at the dermoepidermal junction. ${ }^{6}$

\section{Bullous impetigo}

Bullous impetigo is a superficial skin infection encountered most frequently among children. It typically presents with multiple vesicular and bullous lesions with erythematous bases that eventually crust over. The bullous form of impetigo is highly contagious and is caused by an epidermolytic toxin produced most commonly by staphylococci of phage group II at the site of infection. ${ }^{7}$ The condition may be associated with fever, diarrhea and weakness. A diagnosis of bullous impetigo is made clinically, although swabs of the lesions for culture can isolate the causative agent. Local treatment with $2 \%$ mupirocin ointment is sufficient to cure mild infection, but complicated or widespread infection may require oral $\beta$ lactamase-resistant antibiotics. ${ }^{7}$

\section{Dermatitis herpetiformis}

Dermatitis herpetiformis is a subepidermal immunobullous dermatosis that is rarely observed among children. It predominantly affects young adults (mean age 30 years) and is characterized by a granular IgA deposit in the basement membrane zone especially in the papilla. ${ }^{8}$ Cutaneous lesions consist of vesicles arising on erythematous plaques or on skin without plaques. It can be associated with celiac disease, and it responds well to dapsone. ${ }^{8}$ 


\section{Mastocytosis}

Mastocytosis is characterized by an increased number of mast cells with an abnormal growth and accumulation in one or more organs. In children, mastocytosis is usually limited to the skin. The most common form in children is maculopapulous mastocytosis (formerly referred to as urticaria pigmentosa). Bullous mastocytosis may be rarely observed in extensive forms of cutaneous mastocytosis, caused by separation between the epidermis and dermis. A diagnosis of bullous mastocytosis is based on the results of histological and immunohistochemical studies following cutaneous biopsy. ${ }^{9}$

\section{Treatment}

Systemic corticosteroid therapy (prednisone 1-2 mg/kg daily) has been the usual treatment of choice in childhood bullous pemphigoid. ${ }^{10}$ However, a recent review based on expert interpretation of published reports on the treatment of bullous pemphigoid has concluded that high-dose topical clobetasol propionate should be the first-line treatment. ${ }^{10}$ In our patient, topical therapy with high-potency corticosteroids induced remission.

Corticosteroid-refractory bullous pemphigoid is very rare in children, but in such cases, dapsone, mycophenolate mofetil, cyclosporine, azathioprine, plasmapheresis and rituximab may be good alternatives. ${ }^{11}$ Generally, infants and children with bullous pemphigoid have a good prognosis, and remission is achieved within several weeks to a few months. The response to treatment is rapid and ranges from a few days to several months; relapses are very rare. ${ }^{12}$ Secondary cutaneous infections or sepsis may occur, especially in children with widespread involvement. Complications related to the use of systemic or extensive local corticosteroids are also possible.
In conclusion, a diagnosis of childhood bullous pemphigoid should be considered in any infant or child presenting with tense bullae, especially on the palms and soles.

This article has been peer reviewed.

Competing interests: None declared.

\section{REFERENCES}

1. Waisbourd-Zinman $\mathrm{O}$, Ben-Amitai $\mathrm{D}$, Cohen $\mathrm{AD}$, et al. Bullous pemphigoid in infancy: clinical and epidemiologic characteristics. J Am Acad Dermatol 2008;58:41-8.

2. Garcia-Doval I, Mayo E, Nogueira Farina J, et al. Bullous pemphigoid triggered by influenza vaccination? Ecological study in Galicia, Spain. Br J Dermatol 2006; 155:820-3.

3. Toyama T, Nakamura K, Kuramochi A, et al. Two cases of childhood bullous pemphigoid. Eur J Dermatol 2009;19:368-71.

4. Nemeth AJ, Klein AD, Gould EW, et al. Childhood bullous pemphigoid: clinical and immunologic features, treatment and prognosis. Arch Dermatol 1991;127:378-86.

5. Martinez-De Pablo MI, González-Enseñat MA, Vicente A, et al. Childhood bullous pemphigoid: clinical and immunological findings in a series of 4 cases. Arch Dermatol 2007; 143:215-20.

6. Guide S V, Marinkovich M P. Linear Ig A bullous dermatosis. Clin Dermatol 2001;19:719-27.

7. Al-Hammadi H, Dermacase. Bullous impetigo. Can Fam Physician. 2008;54:193-7.

8. Templet JT, Welsh JP, Cusack CA. Childhood dermatitis herpetiformis: a case report and review of the literature. Cutis 2007;80:473-6.

9. Heide R, Beishuizen A, De Groot H, et al.; Dutch National Mastocytosis Work Group. Mastocytosis in children: a protocol for management. Pediatr Dermatol 2008;25:493-500.

10. Roujeau JC, Ingen-Housz-Oro S, Leroux $\mathrm{C}$, et al. Treatment of bullous pemphigoid and pemphigus. The French experience, 2009 update. G Ital Dermatol Venereol 2009;144:333-8.

11. Schulze J, Bader P, Henke U, et al. Severe bullous pemphigoid in an infant - successful treatment with rituximab. Pediatr Dermatol 2008;25:462-5.

12. Sáenz AM, González F, Cirocco A, et al. Childhood bullous pemphigoid: a case report and 10-year follow up. Int J Dermatol 2007;46:508-10.

Correspondence to: Dr. Aida Khaled, Blvd. 9 Avril, Tunis,

Tunisia 1006; aida.khaled@rns.tn

\section{Atacand}

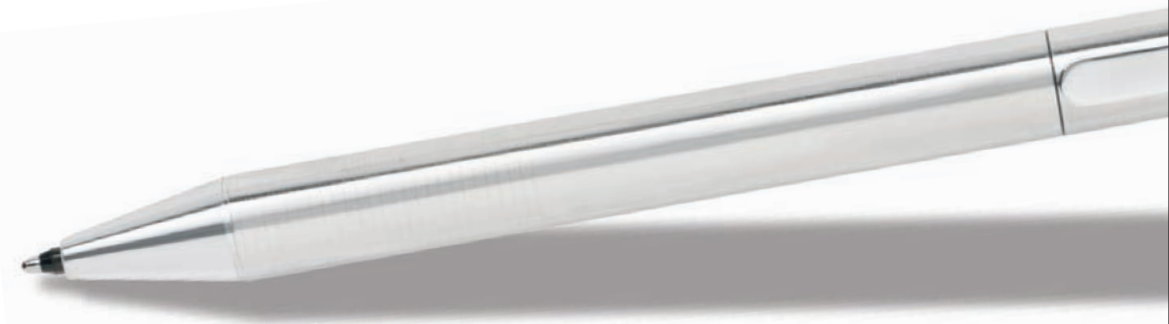

Atacand 